Doi: HTTPS://DOI.ORG/10.23910/IJEP/2018.5.3.0251

\title{
Effect of Vase Solution on Value Addition and Vase-life of Tinted Tuberose (Polianthes tuberosa L.) cv. Prajwal
}

\author{
Sweta Kumari ${ }^{1}$ and Prahlad Deb ${ }^{2}$ \\ ${ }^{1}$ Dept. of Floriculture and Landscape Architecture, BCKV, Mohanpur, Nadia, West Bengal (741 252), India \\ ${ }^{2}$ Dept. of Horticulture \& Post-Harvest Technology instead Sriniketan, West Bengal (731 235), India
}

\section{Corresponding Author}

Sweta Kumari

e-mail: sweta.sharmaa89@gmail.com

\author{
Article History \\ Article ID: IJEP0251 \\ Received in $12^{\text {th }}$ June, 2018 \\ Received in revised form $19^{\text {th }}$ July, 2018 \\ Accepted in final form $17^{\text {th }}$ August, 2018
}

\begin{abstract}
Flower craft is one of the most flourish and dynamic enterprises in today's world. Tinting or artificial colouring of tuberose may be a potential value addition venture. Tuberose (Polianthes tuberosa L.) is a popular cut flower having white coloured fragrant blooms. Postharvest losses in many cut are estimated to be as high as $40 \%$ in the absence of floral preservatives. Blockage of vascular bundles due to microorganism proliferation is one of the reasons for cut flower postharvest losses. Therefore in this experiment effect of different biocides on kind and proliferation of microbes in vase solution of tinted tuberose were studied. In present experiment five different food dyes were used as tinting agent likes Blue, Apple green, Lemon yellow, Orange red and Rose pink at a concentration of $1 \%$. Therefore an inspection was carried out to study the effect of different biocides (Calcium hypochlorite solution of $750 \mathrm{ppm}$, Sodium hypochlorite solution of $750 \mathrm{ppm}$, Benzyl adenine $450 \mathrm{ppm}$ and Naphthalene acetic acid $250 \mathrm{ppm}$ ) on value addition and vase-life of tinted tuberose cv. Prajwal. In which flowers remained maximum fresh with higher vase-life in Lemon yellow followed by Rose pink and Apple green. Results suggest that among all chemicals Calcium hypochlorite (750 ppm) was best followed by Sodium hypochlorite (750 ppm), Benzyl adenine (450 ppm) and Naphthalene acetic acid (250 ppm).
\end{abstract}

Keywords: Biocide, microbial proliferation, vase solution

\section{Introduction}

Flowers are the wonderful creations of the nature and are one of the most beautiful gifts of nature and they are integral part of human life. In this craft, value addition and postharvest handling methods of cut flowers is a captivating and trilling craft gaining importance now-a-days. The tuberose (Polianthes tuberosa L.) is a half-hardy, perennial, bulbous plant belongs to family Agavaceae. Tinting is an important value addition technique in flower crops where colour pigments are absent or light or dull. Colouring inflorescences with edible dyes enhance the visual appeal of these flowers, increase their economic value and aesthetic beautification. For decorative purpose where a particular colour is desired, tinting of white flower is an easy way of obtaining the colour of interest whereas, certified synthetic food colours are less expensive, less hazardous and don't impart an intense and uniform colour to the tinted flowers. Tinting techniques has already been experimented in tuberose (Sambandhamurthy and Appavu., 1980; Kumar et al., 2003; Mekala et al., 2012) and Lady's Lace (Patil and Dhaduk., 2008).
The short vase-life of many cut flowers continues to pose a challenge to the floral craft. Short vase-life of tuberose can be associated with, among other factors, unfavourable water balance i.e. the difference between water uptake and loss. Calcium hypochlorite is an inorganic compound with formula $\mathrm{Ca}(\mathrm{ClO})_{2}$. As a mixture with lime and calcium chloride, it is marketed as chlorine powder or bleaching powder for water treatment and as a bleaching agent. This compound is relatively stable and has greater available chlorine than sodium hypochlorite (liquid bleach). Sodium hypochlorite is practically and chemically distinct from chlorine, but may be converted into it by the addition of acid. Calcium hypochlorite and sodium hypochlorite are very good vase preservative. It was previously used by (Jowkar, 2007). 1-Naphthaleneacetic acid, commonly abbreviated NAA is an organic compound with the formula $\mathrm{C}_{10} \mathrm{H}_{7} \mathrm{CH}_{2} \mathrm{CO}_{2} \mathrm{H}$. NAA is a plant hormone in the auxin family and is an ingredient in many commercial postharvest horticultural products. It is also a rooting agent and used for vegetative propagation of plant from stem and leaf cutting (Dimitrios et al., 2008; Saifuddin., 2009). 6-Benzyladenine is a synthetic cytokinin that stimulates cell division in plants. 
Among other actions, it spurs plant growth, sets blossoms, and improves fruit quality. Benzyl adenine may have delayed primary floret opening because cytokinin and gibberellins are documented to delaying senescence of cut flowers (Salisbury and Ross, 1986). Blockage of vascular bundles due to microorganism proliferation is one of the reasons for cut flower postharvest losses. Microbial contamination of cut tuberose vase solution was mostly due to bacteria such as Streptomyces, negative gram Bacillus, negative gram Cocci. In which Aspergillus and yeasts were the most spread microorganisms. Many post-harvest procedures for cut flowers involve use of various compounds and technologies that inhibit the effects of ethylene reduce respiration or maintain better water relations. Therefore an investigation is carried out to study the effect of different biocides (Calcium hypochlorite, Sodium hypochlorite, Naphthalene acetic acid and Benzyl adenine) on value addition and vase life of tinted tuberose cv. Prajwal.

\section{Materials and Methods}

The site, where experiment was carried out in the departmental laboratory of Department of Horticulture \& Post-Harvest Technology, Palli Siksha Bhavana, Visva-Bharti, Sriniketan during December 2014 to January 2015. Five different food dyes were used as tinting agent in the experiment all in powder form viz, Blue $\left(P_{1}\right)$, Apple green $\left(P_{2}\right)$, Lemon Yellow $\left(P_{3}\right)$, Orange red $\left(P_{5}\right)$ and Rose pink $\left(P_{6}\right)$ respectively in place of Blue, Apple green, Lemon Yellow, Orange red and Rose pink respectively, at a concentration of $1 \%$ for all. Four different vase solution like $S_{1}$ (Calcium hypochlorite 750 ppm), $\mathrm{S}_{2}$ (Sodium hypochlorite 750 ppm), $\mathrm{S}_{3}$ (Benzyl adenine $450 \mathrm{ppm}$ ) and $\mathrm{S}_{4}$ (Naphthalene acetic acid 250 ppm) were used. There were 21 different treatments with three replications in each. Different observation like colour solution uptake, amount of water absorbed during tinting, effect of colour and different vase solution on spike weight, number of flower open, number of flower drop, flower length and flower diameter( $3^{\text {rd }}$ number of flower) were taken. Flowers were harvested in the morning between 8.00 and 9.00 am. Flowers were harvested from farmer field with 2-3 flowers open in each spike. Immediately after harvest, the cut ends of the flower stalks were immersed in water. To prepare $1 \%$ of colour solution powder of Blue, Apple green, Lemon yellow, Orange red and Rose pink colour of $1 \mathrm{~g}$ is mixed in $100 \mathrm{ml}$ of filtrated water. The uniform spikes with $60 \mathrm{~cm}$ stalk length with 2-3 florets opening with 3 spikes were put in conical flask containing $100 \mathrm{ml}$ of edible dye solutions. Spikes were removed from colour solution after 6 hours immediately after it put in vase solution. Note: Benzyl adenine was dissolve in ethanol and Naphthalene acetic acid in $\mathrm{NaOH}$. First dissolve benzyl adenine in ethanol and Naphthalene acetic acid in $\mathrm{NaOH}$ after that mixed in water. Flasks of $500 \mathrm{ml}$ capacity were used. $200 \mathrm{ml}$ of filtered water used for vase solution. The data were analyzed factorial completely randomized block with two factor colour and solution.

\section{Results and Discussion}

3.1. Amount of colour solution and vase solution absorbed by tuberose flower ( $\mathrm{ml}$ spike-1)

The different food dyes solutions showed the significant difference for amount of colour solution absorption (Table 1) in zero days. The maximum amount of colour solution absorbed in Rose pink (21.84) followed by Apple green (10.73), Blue (10.63), Lemon yellow (10.13) food dyes, whereas minimum amount of colour solution absorbed in Orange red (9.69). The colour solution absorption also showed significant difference for the vase solution maximum colour absorption occurs in $S_{1}$ (13.57) followed by $S_{2}(12), S_{3}(12.27)$ and lowest in $S_{4}(11.66)$. In combination of colour and solution shows significant difference in absorption maximum absorption occurs in $P_{6} S_{1}$ i.e. 26.3 followed by $P_{6} S_{2}$ (23.3), $P_{6} S_{3}(21.1), P_{6} S_{4}(16.7), P_{1} S_{2}$ (11.2), $P_{2} S_{3}(11), P_{2} S_{2}(10.9), P_{1} S_{1}(10.6), P_{2} S_{1}(10.6), P_{1} S_{3}(10.5)$, $P_{2} S_{4}(10.4), P_{3} S_{1}(10.3), P_{1} S_{4}(10.2), P_{5} S_{1}(10.1), P_{3} S_{3}(9.8), P_{3} S_{2}$ (9.6), $P_{5} S_{2}$ (9.6) and minimum 9 in $P_{5} S_{3}$.

The data recorded on water uptake by tuberose flower was significantly affected by different biocide solutions. Control recorded significantly highest water absorption (14.55) followed by Apple green (7.13), Rose pink (6.88), Lemon yellow (6.02), Blue (5.13) and least in Orange red (4.94) on first day. Generally the water absorption decreased from second days to seven days during vase solution. The interaction effect of water uptake between colour and vase solution also showed significant difference. The treatment $S_{1}$ recorded significantly highest water uptake on days 1, 2, 3, 4, 5, 6 and 7 (7.44, $3.24,3.40,3.40,1.82$ and 0.88 respectively) followed by $S_{2}$ $(5.99,3.44,3.53,3.53,1.76,1.52$ and 1.19 respectively), $\mathrm{S}_{3}(5.33,3.40,3.48,3.48,1.47,1.01$ and 0.92 respectively) and significantly lowest water uptake was observed in $\mathrm{S}_{4}$ (5.33, 3.36, 2.62, 2.62, 1.09, 0.92 and 0.19 respectively). In combination of colour and vase solution showed significant difference in absorption maximum absorption occurs in $\mathrm{P}_{2} \mathrm{~S}_{1}$ i.e. 10.4 followed by $P_{6} S_{4}(8.7), P_{3} S_{1}(8.1), P_{5} S_{3}(8.0), P_{6} S_{2}($ 7.8 ), $P_{6} S_{1}(7.3), P_{2} S_{3}(7), P_{1} S_{1}(6.7), P_{2} S_{4}(5.8), P_{2} S_{2}(5.3), P_{3} S_{2}$ (5.3), $P_{1} S_{4}(4.9), P_{1} S_{3}(4.1), P_{6} S_{3}(3.8), P_{5} S_{1}(3.8), P_{3} S_{4}(3.6)$ and minimum in $\mathrm{P}_{5} \mathrm{~S}_{2}(3.3)$ in first day. The highest Water uptake was observed due to effective transportation within the floral stems and reduced stem blockage, which was supported by the findings of Marousky (1969) in cut roses. The findings of De Jong (1978); Mayak (1981) and Dumitras et al. (2002) in different cut flowers and Pand and Santhosh (2004) also reported similar observations in cut gladiolus spike.

\subsection{Effect of vase solution on spike weight ( $g$ spike ${ }^{-1}$ ) of tinted tuberose}

Effect of solution on spike weight of tinted tuberose was 


\begin{tabular}{|c|c|c|c|c|c|c|c|c|}
\hline Treatments & 0 day & 1 Days & 2 Days & 3 Days & 4 Days & 5 Days & 6 Days & 7 Days \\
\hline \multicolumn{9}{|l|}{ Colour } \\
\hline$P_{0}$ & - & 14.55 & 2.65 & 2.21 & 1.55 & 1.50 & 1.46 & 1.30 \\
\hline$P_{1}$ & 10.63 & 5.13 & 4.33 & 3.07 & 3.07 & 1.60 & 1.59 & 1.18 \\
\hline$P_{2}$ & 10.73 & 7.13 & 4.19 & 2.85 & 2.85 & 2.55 & 2.06 & 1.44 \\
\hline$P_{3}$ & 10.13 & 6.02 & 4.02 & 1.83 & 1.83 & 1.44 & 1.00 & 0.17 \\
\hline$P_{5}$ & 9.69 & 4.94 & 2.00 & 2.57 & 2.57 & 0.00 & 0.00 & 0.00 \\
\hline $\mathrm{P}_{6}$ & 21.84 & 6.88 & 2.27 & 5.97 & 5.97 & 2.81 & 1.93 & 1.21 \\
\hline SEd \pm & 0.54 & 0.75 & 0.60 & 0.61 & 0.61 & 0.49 & 0.37 & 0.28 \\
\hline$C D(p=0.05)$ & 1.08 & 1.51 & 1.22 & 1.22 & 1.22 & 1.00 & 0.75 & 0.57 \\
\hline \multicolumn{9}{|l|}{ Solution } \\
\hline $\mathrm{S}_{1}$ & 13.57 & 7.44 & 3.24 & 3.40 & 3.40 & 2.39 & 1.82 & 0.88 \\
\hline $\mathrm{S}_{2}$ & 12.90 & 5.99 & 3.44 & 3.53 & 3.53 & 1.76 & 1.52 & 1.19 \\
\hline $\mathrm{S}_{3}$ & 12.27 & 5.33 & 3.40 & 3.48 & 3.48 & 1.47 & 1.01 & 0.92 \\
\hline $\mathrm{S}_{4}$ & 11.66 & 5.33 & 3.36 & 2.62 & 2.62 & 1.09 & 0.92 & 0.19 \\
\hline SEd \pm & 0.61 & 0.84 & 0.67 & 0.67 & 0.67 & 0.44 & 0.33 & 0.32 \\
\hline$C D(p=0.05)$ & 1.22 & 1.69 & 1.36 & 1.36 & 1.36 & 0.89 & 0.67 & 0.64 \\
\hline \multicolumn{9}{|c|}{ Colour $\times$ Solution } \\
\hline $\mathrm{P}_{1} \mathrm{~S}_{1}$ & 10.6 & 6.7 & 4.0 & 3.2 & 3.2 & 2.3 & 1.9 & 1.3 \\
\hline $\mathrm{P}_{2} \mathrm{~S}_{1}$ & 10.6 & 10.4 & 4.7 & 3.1 & 3.1 & 2.6 & 1.8 & 0.7 \\
\hline $\mathrm{P}_{3} \mathrm{~S}_{1}$ & 10.3 & 8.1 & 4.0 & 1.8 & 1.8 & 1.6 & 1. & 0.7 \\
\hline $\mathrm{P}_{5} \mathrm{~S}_{1}$ & 10.1 & 4.7 & 1.2 & 2.4 & 2.4 & - & - & - \\
\hline $\mathrm{P}_{6} \mathrm{~S}_{1}$ & 26.3 & 7.3 & 2.3 & 6.6 & 6.6 & 5.5 & 4.3 & 1.8 \\
\hline $\mathrm{P}_{1} \mathrm{~S}_{2}$ & 11.2 & 4.9 & 3.7 & 5.1 & 5.1 & 2.0 & 3.1 & 2.9 \\
\hline $\mathrm{P}_{2} \mathrm{~S}_{2}$ & 10.9 & 5.3 & 5.3 & 3.3 & 3.3 & 2.2 & 1.5 & 1.5 \\
\hline $\mathrm{P}_{3} \mathrm{~S}_{2}$ & 9.6 & 5.3 & 3.7 & 1.8 & 1.8 & 1.8 & 1.0 & - \\
\hline $\mathrm{P}_{5} \mathrm{~S}_{2}$ & 9.6 & 3.3 & 2.6 & 3.0 & 3.0 & - & - & - \\
\hline $\mathrm{P}_{6} \mathrm{~S}_{2}$ & 23.3 & 7.8 & 2.0 & 4.4 & 4.4 & 2.8 & 1.9 & 1.6 \\
\hline $\mathrm{P}_{1} \mathrm{~S}_{3}$ & 10.5 & 4.1 & 4.6 & 2.4 & 2.4 & 1.1 & 0.5 & 0.6 \\
\hline $\mathrm{P}_{2} \mathrm{~S}_{3}$ & 11.0 & 7.0 & 3.5 & 3.3 & 3.3 & 3.5 & 3.1 & 3.6 \\
\hline $\mathrm{P}_{3} \mathrm{~S}_{3}$ & 9.8 & 7.1 & 4.2 & 1.3 & 1.3 & 1.5 & 1.0 & - \\
\hline $\mathrm{P}_{5} \mathrm{~S}_{3}$ & 9.0 & 8.0 & 1.8 & 1.3 & 1.3 & - & - & - \\
\hline $\mathrm{P}_{6} \mathrm{~S}_{3}$ & 21.1 & 3.8 & 2.9 & 9.0 & 9.0 & 1.2 & 0.5 & 0.5 \\
\hline $\mathrm{P}_{1} \mathrm{~S}_{4}$ & 10.2 & 4.9 & 5.0 & 1.6 & 1.6 & 1.0 & 0.9 & - \\
\hline $\mathrm{P}_{2} \mathrm{~S}_{4}$ & 10.4 & 5.8 & 3.2 & 1.7 & 1.7 & 1.9 & 1.9 & - \\
\hline $\mathrm{P}_{3} \mathrm{~S}_{4}$ & 10.9 & 3.6 & 4.2 & 2.4 & 2.4 & 0.8 & 0.8 & - \\
\hline $\mathrm{P}_{5} \mathrm{~S}_{4}$ & 10.1 & 3.8 & 3.6 & 2.3 & 3.6 & - & - & - \\
\hline $\mathrm{P}_{6} \mathrm{~S}_{4}$ & 16.7 & 8.7 & 3.9 & 2.7 & 3.9 & 1.0 & 1.0 & 1.0 \\
\hline SEd \pm & 1.21 & 1.67 & 1.34 & 1.34 & 1.34 & 0.98 & 0.74 & 0.63 \\
\hline $\mathrm{CD}(p=0.05 \%)$ & 2.43 & 3.38 & 2.71 & 2.71 & 2.71 & 2.0 & 1.5 & 1.27 \\
\hline
\end{tabular}

$\mathrm{P}_{0}$ : White; $\mathrm{P}_{1}$ : Blue; $\mathrm{P}_{2}$ : Apple green; $\mathrm{P}_{3}$ : Lemon yellow; $\mathrm{P}_{5}$ : Orange red; $\mathrm{P}_{6}$ : Rose pink; $\mathrm{S}_{1}$ : Calcium hypochlorite $750 \mathrm{ppm} ; \mathrm{S}_{2}$ : Sodium hypochlorite 750 ppm; $\mathrm{S}_{3}$ : Benzyl adenine 450 ppm; $\mathrm{S}_{4}$ : Naphthalene acetic acid 250 ppm; In table "-" denote that treatment was lost 


\begin{tabular}{|c|c|c|c|c|c|c|c|c|}
\hline Treatments & 0 day & 1 Days & 2 Days & 3 Days & 4 Days & 5 Days & 6 Days & 7 Days \\
\hline \multicolumn{9}{|l|}{ Colour } \\
\hline$P_{0}$ & 39.71 & 40.77 & 37.74 & 35.88 & 27.36 & 31.65 & 17.33 & 16.75 \\
\hline$P_{1}$ & 38.33 & 42.44 & 38.77 & 33.66 & 24.10 & 22.72 & 19.26 & 18.25 \\
\hline $\mathrm{P}_{2}$ & 39.32 & 44.37 & 41.29 & 34.10 & 26.68 & 22.48 & 20.00 & 18.92 \\
\hline$P_{3}$ & 39.29 & 43.80 & 38.86 & 32.55 & 25.67 & 21.80 & 14.08 & 17.54 \\
\hline $\mathrm{P}_{5}$ & 39.31 & 42.16 & 39.58 & 34.23 & 26.05 & 21.57 & 4.67 & 0.00 \\
\hline$P_{6}$ & 38.81 & 42.11 & 39.07 & 36.63 & 31.53 & 24.88 & 22.67 & 20.88 \\
\hline SEd \pm & 0.44 & 0.49 & 1.35 & 0.95 & 0.93 & 1.01 & 0.51 & 1.16 \\
\hline$C D(p=0.05)$ & NS & 1.01 & NS & NS & 1.89 & NS & 1.03 & 2.36 \\
\hline \multicolumn{9}{|l|}{ Solution } \\
\hline $\mathrm{S}_{1}$ & 36.53 & 43.06 & 40.34 & 33.95 & 27.64 & 22.92 & 16.81 & 14.80 \\
\hline $\mathrm{S}_{2}$ & 39.37 & 41.77 & 38.44 & 33.79 & 25.57 & 22.55 & 15.79 & 15.03 \\
\hline $\mathrm{S}_{3}$ & 39.51 & 43.10 & 38.93 & 34.27 & 25.46 & 21.59 & 15.67 & 14.67 \\
\hline $\mathrm{S}_{4}$ & 40.65 & 43.96 & 40.34 & 34.93 & 28.54 & 23.69 & 16.27 & 15.93 \\
\hline SEd \pm & 0.48 & 0.56 & 1.52 & 1.06 & 1.04 & 1.13 & 0.57 & 1.04 \\
\hline$C D(p=0.05)$ & 0.98 & 1.13 & NS & 2.14 & 2.11 & 2.28 & NS & NS \\
\hline \multicolumn{9}{|c|}{ Colour×Solution } \\
\hline $\mathrm{P}_{1} \mathrm{~S}_{1}$ & 35.1 & 42.2 & 38.9 & 34.2 & 23.4 & 22.1 & 19.4 & 18.7 \\
\hline $\mathrm{P}_{2} \mathrm{~S}_{1}$ & 36.1 & 45.2 & 44.6 & 36.5 & 29.9 & 24.0 & 20.3 & 20.0 \\
\hline$P_{3} S_{1}$ & 37.4 & 46.7 & 40.9 & 30.3 & 27.4 & 22.2 & 19.3 & 18.0 \\
\hline $\mathrm{P}_{5} \mathrm{~S}_{1}$ & 36.1 & 39.3 & 37.6 & 31.7 & 23.7 & 20.6 & - & - \\
\hline$P_{6} S_{1}$ & 37.9 & 41.8 & 39.6 & 37.0 & 33.8 & 25.7 & 25.0 & 23.0 \\
\hline $\mathrm{P}_{1} \mathrm{~S}_{2}$ & 40.5 & 41.8 & 40.7 & 35.3 & 24.5 & 23.8 & 19.5 & 18.5 \\
\hline $\mathrm{P}_{2} \mathrm{~S}_{2}$ & 40.0 & 42.0 & 42.1 & 35.4 & 26.5 & 23.6 & 19.8 & 18.7 \\
\hline $\mathrm{P}_{3} \mathrm{~S}_{2}$ & 39.0 & 41.9 & 36.9 & 32.6 & 24.0 & 21.8 & 18.7 & 17.5 \\
\hline$P_{5} S_{2}$ & 38.5 & 41.7 & 34.8 & 29.8 & 24.0 & 20.9 & - & - \\
\hline $\mathrm{P}_{6} \mathrm{~S}_{2}$ & 38.8 & 41.5 & 37.7 & 35.8 & 28.9 & 22.7 & 21.0 & 19.5 \\
\hline $\mathrm{P}_{1} \mathrm{~S}_{3}$ & 39.3 & 42.2 & 37.7 & 34.2 & 25.2 & 22.3 & 18.5 & 17.5 \\
\hline $\mathrm{P}_{2} \mathrm{~S}_{3}$ & 40.0 & 43.5 & 39.4 & 30.4 & 25.7 & 20.4 & 20.2 & 18.8 \\
\hline $\mathrm{P}_{3} \mathrm{~S}_{3}$ & 39.2 & 44.1 & 38.9 & 33.5 & 24.2 & 22.1 & 19.0 & 17.8 \\
\hline $\mathrm{P}_{5} \mathrm{~S}_{3}$ & 40.8 & 44.3 & 41.2 & 37.2 & 22.2 & 20.3 & - & - \\
\hline $\mathrm{P}_{6} \mathrm{~S}_{3}$ & 38.3 & 41.5 & 37.4 & 36.0 & 30.0 & 22.8 & 20.7 & 19.2 \\
\hline $\mathrm{P}_{1} \mathrm{~S}_{4}$ & 38.5 & 43.6 & 37.7 & 30.8 & 23.3 & 22.7 & 19.7 & 18.3 \\
\hline $\mathrm{P}_{2} \mathrm{~S}_{4}$ & 41.2 & 44.5 & 39.0 & 34.1 & 24.7 & 22.0 & 19.7 & 18.2 \\
\hline $\mathrm{P}_{3} \mathrm{~S}_{4}$ & 41.6 & 44.8 & 38.8 & 33.8 & 27.0 & 21.0 & 18.0 & 16.8 \\
\hline $\mathrm{P}_{5} \mathrm{~S}_{4}$ & 41.8 & 43.4 & 44.6 & 38.3 & 34.3 & 24.5 & - & - \\
\hline $\mathrm{P}_{6} \mathrm{~S}_{4}$ & 40.2 & 43.6 & 41.6 & 37.7 & 33.4 & 28.3 & 24.0 & 21.8 \\
\hline SEd \pm & 2.85 & 2.95 & 3.03 & 4.23 & 4.21 & 4.55 & 2.29 & 2.33 \\
\hline $\mathrm{CD}(p=0.05 \%)$ & 1.97 & 2.25 & NS & 4.28 & 4.22 & NS & 2.29 & NS \\
\hline
\end{tabular}

mostly non-significant up to $3^{\text {rd }}$ days (Table 2). The minimum maximum weight gained by $P_{2}(44.37$ ) on first day. Spike flower weight observed at par with $P_{0}$ (40.77) while weight showed the significant difference for food dyes and 
solution treatments on first day to seventh days. The highest spike weight recorded in treatment $S_{4}$ on day $1,2,3,4,5,6$ and 7 (43.96, 40.34, 34.93, 28.54, 23.69, 16.27 and 15.93 respectively) followed by $S_{3}(43.10,38.93,34.27,25.46$, $21.59,15.67$ and 14.67 respectively), $S_{2}(41.77,38.44,33.79$, $25.57,22.55,15.79$ and 15.03 respectively) and significantly lowest spike weight observed in $\mathrm{S}_{1}(43.06,40.34,33.95,27.64$, 22.92, 16.81 and 14.80 respectively). This might be due to BA at all concentrations decreased the rate of leaf nitrogen degradation of Alstroemeria cut flowers during the study period compared to the control. Cytokinins had been reported to delay senescence by retarding the rate of breakdown of proteins rather than enhancing the rate of protein synthesis (Sacher, 1973). Richmond and Lang (1957) reported that kinetin (cytokinin) prevented accelerated protein loss that was typical to detached leaves.

\subsection{Effect of vase solution on number of flower open and drop tinted tuberose (flowers spike-1)}

Number of flower open per day showed the significant difference for different food dyes from first to third days, where the maximum number of florets opened in Rose pink was 7.08 and minimum flower opened in Blue was 5.0 (Table 3) for first day. Solution showed significant difference for opening of flowers the highest number recorded in treatment $\mathrm{S}_{3}$ significantly highest on days $1,2,3$ and $4(2.27,6.20,7.20$ and 9.07 respectively) followed by $\mathrm{S}_{4}$ on days $1,2,3$ and 4 $\left(1.73,5.47,7.13\right.$ and 7.73 respectively). $S_{2}(2.07,5.67,6.93$ and 7.47 respectively) and significantly lowest flower opening observed in $S_{1}(1.40,5.27,7.07$ and 7.27 respectively). While in combination of solution and food dye maximum flower opening occurred in $\mathrm{P}_{6} \mathrm{~S}_{2}$ was 8.3 and minimum for $\mathrm{P}_{5} \mathrm{~S}_{4}$ was 4 for first day.

In different colours minimum flower dropping occurred in $\mathrm{P}_{1}$ was 1.75 and maximum flower dropping occurred in (Table 3) $P_{5}$ that was 5.33. In combination minimum of $P_{3} S_{4}$, flower dropping was 1 on first day and maximum flower dropping occurs in $\mathrm{P}_{3} \mathrm{~S}_{4}$ that was 6.7. Number of flower dropping per day showed the significant difference for different food dyes from fourth to seventh days. Minimum number of florets was dropped in control and Blue (0.89 flowers/spike) whereas maximum number of florets was dropped in $5^{\text {th }}$ days on Orange red (6.56 flower spike ${ }^{-1}$ in Table 3 ) on $4^{\text {th }}$ days. The maximum number of dropping of flowers recorded in solution $\mathrm{S}_{4}$ significantly highest on days 4, 5, 6 and 7 (3.47, 10.47, 11.73 and 13.33 respectively) followed by $S_{3}$ in days $4,5,6$ and 7 (3.40, 9.27, 12.27 and 14.47 respectively), $\mathrm{S}_{2}$ (2.07, 11.33 and 10.20 and 7.47 respectively) significantly lowest flower dropping was observed in $S_{1}(3.60,9.60,8.87$, and 13.60 respectively).

\subsection{Flower length and flower diameter $(\mathrm{cm})$}

The flower length of tinted tuberose spikes was seen the significant difference for different food dyes from fourth day onwards (Table 4). Flower length was an important quality parameter when flowers were kept for interior decoration it made the environment pleasant. The maximum flower length was Apple green was $5.42 \mathrm{~cm}$ and minimum for pink rose was $5.18 \mathrm{~cm}$. The maximum flowers length recorded on first day for vase solution $\mathrm{S}_{1}, \mathrm{~S}_{2}, \mathrm{~S}_{4}$ and $\mathrm{S}_{3}$ were $4.55,4.30,4.29$ and 4.28 $\mathrm{cm}$ respectively, followed by second days maximum flower length for $\mathrm{S}_{1}, \mathrm{~S}_{3}, \mathrm{~S}_{2}$ and $\mathrm{S}_{4}$ were $5.42,5.33,5.31$ and $5.28 \mathrm{~cm}$ respectively, third day $S_{4}, S_{1}, S_{3}$ and $S_{2}(5.03,4.98,4.98$ and 4.95 $\mathrm{cm}$ respectively) and on fourth days maximum flower length in $\mathrm{S}_{3}, \mathrm{~S}_{4}, \mathrm{~S}_{1}$ and $\mathrm{S}_{2}(4.56,4.42,4.14$ and $4.12 \mathrm{~cm}$ respectively). The interaction effect of food dyes and solution saw the significant difference for flower length (Table 4).

Flower diameter was increases first two days onwards it was decreases maximum flower diameter was for $P_{3}(4.32 \mathrm{~cm})$ and minimum flower diameter was for $P_{5}(4.24 \mathrm{~cm})$. The solution maximum flowers diameter recorded first days were $S_{1}, S_{2}, S_{3}$ and $S_{4}(3.61,3.58,3.52$ and $3.52 \mathrm{~cm}$ respectively) followed by second day maximum flower diameter were $\mathrm{S}_{1}, \mathrm{~S}_{4}, \mathrm{~S}_{3}$ and $\mathrm{S}_{2}$ $\left(4.30,4.27,4.24\right.$ and $4.20 \mathrm{~cm}$ respectively), third day $S_{1}, S_{3}, S_{4}$ and $\mathrm{S}_{2}(3.94,3.88,3.86$ and $3.82 \mathrm{~cm}$ respectively) and on fourth days maximum flower diameter were $\mathrm{S}_{2}, \mathrm{~S}_{1}, \mathrm{~S}_{3}$ and $\mathrm{S}_{4}(3.35$, $3.20,3.11$ and $2.82 \mathrm{~cm}$ respectively). In combination of colour and solution maximum flower diameter for combination $P_{1} S_{1}$ and $\mathrm{P}_{3} \mathrm{~S}_{3}$ were $4.5 \mathrm{~cm}$ and minimum for $\mathrm{P}_{5} \mathrm{~S}_{1}$ and $\mathrm{P}_{5} \mathrm{~S}_{3}$ were $4.1 \mathrm{~cm}$ (first days).

Lastly it could be concluded that floret length and diameter increases first 2 days after that it decreases. The similar results were observed by Kumar et al. (2003) on postharvest quality of tuberose spikes as affected by colouring agents and storage and Talukdar et al. (2011) on effect of pulsing and different holding solutions on flower quality and vase life of tuberose (Polianthes tuberosa L.) cv. Calcutta Double.

\subsection{Vase-life of tinted flowers (Days)}

Vase-life of tuberose cut spikes showed significant difference for different vase preservatives of food dyes treatments. Maximum vase-life was recorded for control, whereas minimum vase-life was found in sodium hypochlorite 750 concentration. From table number ( 1 and 2, 3 and 4) it was clear that maximum vase-life recorded for the for vase preservatives of calcium hypochlorite (7 days) whereas minimum vase-life was found in Naphthalene acetic acid. The reason being that water uptake may be the important factor in imprving the length of vase-life of cut flower (Halevy and Mayank., 1979). As the leaves on flower transpire water was dawn up through the xylem. If the process was impeded by a vascular blockage and accelarated by incresesd stomatal opening then transpiration exceed uptake and water deficiency will occur (Van Doorn., 1997). So, solute like calcium hypochlorite was added to vase solutions could decrease 


\begin{tabular}{|c|c|c|c|c|c|c|c|c|}
\hline Treatments & 0 day & 1 Days & 2 Days & 3 Days & 4 Days & 5 Days & 6 Days & 7 Days \\
\hline \multicolumn{9}{|l|}{ Colour } \\
\hline $\mathrm{P}_{0}$ & 1.80 & 5.12 & 5.66 & 6.21 & 3 & 8.66 & 12 & 18.33 \\
\hline$P_{1}$ & 1.83 & 5.00 & 6.92 & 6.42 & 1.75 & 8.92 & 12.58 & 17.92 \\
\hline $\mathrm{P}_{2}$ & 1.67 & 5.50 & 6.83 & 6.50 & 2.50 & 7.50 & 14.17 & 16.83 \\
\hline$P_{3}$ & 1.75 & 5.33 & 6.67 & 6.08 & 3.17 & 6.75 & 14.50 & 15.50 \\
\hline$P_{5}$ & 2.00 & 5.33 & 7.33 & 9.92 & 5.33 & 19.25 & - & - \\
\hline $\mathrm{P}_{6}$ & 2.08 & 7.08 & 7.67 & 10.50 & 2.92 & 8.42 & 12.58 & 15.50 \\
\hline SEd \pm & 0.25 & 0.33 & 0.43 & 0.54 & 0.38 & 1.16 & 0.52 & 1.28 \\
\hline$C D(p=0.05)$ & 0.50 & 0.66 & 0.86 & 1.08 & 0.78 & 1.16 & 1.05 & 2.60 \\
\hline \multicolumn{9}{|l|}{ Solution } \\
\hline $\mathrm{S}_{1}$ & 1.40 & 5.27 & 7.07 & 7.27 & 3.60 & 9.60 & 8.87 & 13.60 \\
\hline $\mathrm{S}_{2}$ & 2.07 & 5.67 & 6.93 & 7.47 & 2.07 & 11.33 & 10.20 & 14.47 \\
\hline $\mathrm{S}_{3}$ & 2.27 & 6.20 & 7.20 & 9.07 & 3.40 & 9.27 & 12.27 & 11.20 \\
\hline $\mathrm{S}_{4}$ & 1.73 & 5.47 & 7.13 & 7.73 & 3.47 & 10.47 & 11.73 & 13.33 \\
\hline SEd \pm & 0.22 & 0.36 & 0.47 & 0.60 & 0.43 & 0.51 & 0.46 & 1.15 \\
\hline $\mathrm{CD}(p=0.05)$ & 0.45 & 0.74 & 0.96 & 1.22 & 0.87 & 1.03 & 0.94 & 2.33 \\
\hline \multicolumn{9}{|c|}{ ColourxSolution } \\
\hline$P_{1} S_{1}$ & 1.7 & 5.7 & 7.3 & 8.3 & 2.7 & 10.0 & 11.3 & 15.7 \\
\hline $\mathrm{P}_{2} \mathrm{~S}_{1}$ & 1.0 & 5.0 & 7.7 & 8.0 & 3.3 & 9.0 & 16.0 & 19.3 \\
\hline $\mathrm{P}_{3} \mathrm{~S}_{1}$ & 2.0 & 6.0 & 8.0 & 9.7 & 3.3 & 9.7 & 11.7 & 18.0 \\
\hline$P_{5} S_{1}$ & 2.0 & 5.7 & 4.0 & 8.0 & 5.0 & 18.7 & - & - \\
\hline $\mathrm{P}_{6} \mathrm{~S}_{1}$ & 2.0 & 4.0 & 9.0 & 11.3 & 3.7 & 9.3 & 12.0 & 15.0 \\
\hline $\mathrm{P}_{1} \mathrm{~S}_{2}$ & 2.0 & 5.0 & 6.3 & 6.0 & 1.3 & 9.3 & 9.3 & 19.0 \\
\hline $\mathrm{P}_{2} \mathrm{~S}_{2}$ & 2.0 & 5.7 & 6.7 & 7.0 & 3.0 & 6.7 & 7.7 & 19.3 \\
\hline $\mathrm{P}_{3} \mathrm{~S}_{2}$ & 2.0 & 5.3 & 7.0 & 4.0 & 2.7 & 5.3 & 15.7 & 17.0 \\
\hline $\mathrm{P}_{5} \mathrm{~S}_{2}$ & 2.0 & 4.0 & 8.7 & 10.3 & 6.7 & 19.7 & - & - \\
\hline $\mathrm{P}_{6} \mathrm{~S}_{2}$ & 2.3 & 8.3 & 6.0 & 9.0 & 3.3 & 7.0 & 11.7 & 17.0 \\
\hline$P_{1} S_{3}$ & 2.7 & 5.3 & 7.7 & 6.7 & - & 5.7 & 13.7 & 18.0 \\
\hline $\mathrm{P}_{2} \mathrm{~S}_{3}$ & 2.3 & 6.0 & 6.7 & 5.7 & 1.0 & 6.3 & 17.3 & 11.0 \\
\hline $\mathrm{P}_{3} \mathrm{~S}_{3}$ & 2.0 & 4.7 & 5.0 & 4.3 & - & 5.0 & 15.7 & 11.0 \\
\hline $\mathrm{P}_{5} \mathrm{~S}_{3}$ & 2.0 & 7.7 & 8.7 & 10.7 & 7.3 & 20.3 & - & - \\
\hline $\mathrm{P}_{6} \mathrm{~S}_{3}$ & 2.3 & 7.3 & 7.3 & 10.0 & 2.0 & 9.0 & 14.7 & 16.0 \\
\hline $\mathrm{P}_{1} \mathrm{~S}_{4}$ & 1.0 & 4.0 & 6.3 & 4.7 & 3.0 & 10.7 & 16.0 & 19.0 \\
\hline $\mathrm{P}_{2} \mathrm{~S}_{4}$ & 1.3 & 5.3 & 6.3 & 5.3 & 2.7 & 8.0 & 15.7 & 17.7 \\
\hline $\mathrm{P}_{3} \mathrm{~S}_{4}$ & 1.0 & 5.3 & 6.7 & 6.3 & 6.7 & 7.0 & 15.0 & 16.0 \\
\hline$P_{5} S_{4}$ & 2.0 & 4.0 & 8.0 & 10.7 & 2.3 & 18.3 & - & - \\
\hline $\mathrm{P}_{6} \mathrm{~S}_{4}$ & 1.7 & 5.7 & 7.3 & 8.3 & 2.7 & 8.3 & 12.0 & 14.0 \\
\hline SEd \pm & 0.49 & 0.73 & 0.95 & 1.20 & 0.86 & 1.15 & 1.04 & 2.58 \\
\hline $\mathrm{CD}(p=0.05 \%)$ & 0.99 & 1.48 & 1.93 & 2.43 & 1.74 & 2.32 & 2.11 & 5.21 \\
\hline
\end{tabular}




\begin{tabular}{|c|c|c|c|c|c|c|c|c|}
\hline \multirow[t]{3}{*}{ Treatments } & \multicolumn{2}{|c|}{1 Days } & \multicolumn{2}{|c|}{2 Days } & \multicolumn{2}{|c|}{3 Days } & \multicolumn{2}{|c|}{4 Days } \\
\hline & \multicolumn{8}{|c|}{ Colour } \\
\hline & L. & D. & L. & D. & L. & D. & $\mathrm{L}$. & D. \\
\hline$P_{0}$ & 4.26 & 3.50 & 5.37 & 4.17 & 4.78 & 4.17 & 4.41 & 3.10 \\
\hline$P_{1}$ & 4.36 & 3.59 & 5.32 & 4.27 & 5.00 & 4.27 & 4.31 & 3.01 \\
\hline $\mathrm{P}_{2}$ & 4.40 & 3.63 & 5.42 & 4.27 & 5.16 & 4.27 & 4.39 & 2.89 \\
\hline$P_{3}$ & 4.42 & 3.60 & 5.36 & 4.32 & 5.13 & 4.32 & 4.36 & 3.12 \\
\hline$P_{5}$ & 4.37 & 3.37 & 5.34 & 4.14 & 5.12 & 4.14 & 4.45 & 3.33 \\
\hline$P_{6}$ & 4.33 & 3.59 & 5.18 & 4.27 & 4.73 & 4.27 & 3.96 & 3.27 \\
\hline SEd \pm & 4.26 & 0.05 & 5.37 & 0.04 & 4.78 & 0.04 & 4.41 & 0.12 \\
\hline $\mathrm{CD}(p=0.05)$ & 0.03 & NS & 0.04 & 0.08 & 0.04 & 0.08 & 0.06 & 0.24 \\
\hline $\mathrm{S}_{1}$ & 4.55 & 3.61 & 5.42 & 4.30 & 4.98 & 3.20 & 4.14 & 3.20 \\
\hline $\mathrm{S}_{2}$ & 4.30 & 3.58 & 5.31 & 4.20 & 4.95 & 3.35 & 4.12 & 3.35 \\
\hline $\mathrm{S}_{3}$ & 4.28 & 3.52 & 5.33 & 4.24 & 4.98 & 3.11 & 4.56 & 3.11 \\
\hline $\mathrm{S}_{4}$ & 4.29 & 3.52 & 5.28 & 4.27 & 5.03 & 2.82 & 4.42 & 2.82 \\
\hline SEd \pm & 0.04 & 0.05 & 0.04 & 0.04 & 0.04 & 0.05 & 0.06 & 0.13 \\
\hline $\mathrm{CD}(p=0.05)$ & 0.08 & 0.11 & 0.09 & 0.08 & 0.08 & 0.09 & 0.13 & 0.27 \\
\hline $\mathrm{P}_{1} \mathrm{~S}_{1}$ & 4.7 & 3.7 & 5.5 & 4.5 & 5.2 & 4.5 & 4.2 & 3.1 \\
\hline $\mathrm{P}_{2} \mathrm{~S}_{1}$ & 4.6 & 3.7 & 5.5 & 4.3 & 5.1 & 4.3 & 4.1 & 3.2 \\
\hline $\mathrm{P}_{3} \mathrm{~S}_{1}$ & 4.6 & 3.5 & 5.4 & 4.2 & 5.1 & 4.2 & 4.1 & 3.2 \\
\hline $\mathrm{P}_{5} \mathrm{~S}_{1}$ & 4.5 & 3.5 & 5.2 & 4.1 & 4.9 & 4.1 & 3.8 & 3.0 \\
\hline $\mathrm{P}_{6} \mathrm{~S}_{1}$ & 4.3 & 3.7 & 5.4 & 4.4 & 4.7 & 4.4 & 4.4 & 3.6 \\
\hline $\mathrm{P}_{1} \mathrm{~S}_{2}$ & 4.3 & 3.7 & 5.4 & 4.2 & 5.1 & 4.2 & 4.2 & 3.2 \\
\hline $\mathrm{P}_{2} \mathrm{~S}_{2}$ & 4.5 & 3.5 & 5.3 & 4.2 & 5.1 & 4.2 & 4.2 & 2.8 \\
\hline $\mathrm{P}_{3} \mathrm{~S}_{2}$ & 4.2 & 3.6 & 5.3 & 4.2 & 5.1 & 4.2 & 4.2 & 3.5 \\
\hline$P_{5} S_{2}$ & 4.2 & 3.3 & 5.1 & 4.2 & 4.7 & 4.2 & 3.7 & 3.8 \\
\hline $\mathrm{P}_{6} \mathrm{~S}_{2}$ & 4.2 & 3.7 & 5.4 & 4.2 & 4.8 & 4.2 & 4.3 & 3.4 \\
\hline $\mathrm{P}_{1} \mathrm{~S}_{3}$ & 4.2 & 3.6 & 5.4 & 4.2 & 5.1 & 4.2 & 4.6 & 3.0 \\
\hline $\mathrm{P}_{2} \mathrm{~S}_{3}$ & 4.3 & 3.7 & 5.4 & 4.2 & 5.2 & 4.2 & 4.7 & 2.7 \\
\hline $\mathrm{P}_{3} \mathrm{~S}_{3}$ & 4.3 & 3.6 & 5.4 & 4.5 & 5.2 & 4.5 & 4.7 & 2.9 \\
\hline $\mathrm{P}_{5} \mathrm{~S}_{3}$ & 4.2 & 3.2 & 5.2 & 4.1 & 4.7 & 4.1 & 4.5 & 3.7 \\
\hline $\mathrm{P}_{6} \mathrm{~S}_{3}$ & 4.3 & 3.4 & 5.3 & 4.2 & 4.8 & 4.2 & 4.3 & 3.3 \\
\hline $\mathrm{P}_{1} \mathrm{~S}_{4}$ & 4.3 & 3.5 & 5.3 & 4.2 & 5.2 & 4.2 & 4.5 & 2.8 \\
\hline $\mathrm{P}_{2} \mathrm{~S}_{4}$ & 4.3 & 3.6 & 5.2 & 4.4 & 5.1 & 4.4 & 4.5 & 2.8 \\
\hline $\mathrm{P}_{3} \mathrm{~S}_{4}$ & 4.3 & 3.6 & 5.2 & 4.3 & 5.2 & 4.3 & 4.7 & 2.9 \\
\hline $\mathrm{P}_{5} \mathrm{~S}_{4}$ & 4.3 & 3.4 & 5.2 & 4.2 & 4.7 & 4.2 & 3.8 & 2.8 \\
\hline $\mathrm{P}_{6} \mathrm{~S}_{4}$ & 4.3 & 3.5 & 5.4 & 4.2 & 4.9 & 4.2 & 4.6 & 2.8 \\
\hline SEd \pm & 0.07 & 0.11 & 0.09 & 0.08 & 0.08 & 0.08 & 0.13 & 0.27 \\
\hline $\operatorname{CD}(p=0.05 \%)$ & 0.15 & NS & 0.18 & NS & 0.16 & NS & 0.26 & NS \\
\hline
\end{tabular}


transpiration or increase water uptake. So flower remains fresh for more days.

\section{Conclusion}

Tinted spike subjected with different vase solution in which flowers remained maximum fresh with higher vase-life in Lemon yellow followed by Rose pink and Apple green. In different solution calcium hypochlorite was best followed by Sodium hypochlorite, BA then Naphthalene acetic acid on fourth day.

\section{Acknowledgement}

Author acknowledged the support of the Head, Dept. of Horticulture \& Postharvest Technology and Principal, Palli Siksha Bhavana, Visva-Bharti during the course of work.

\section{Reference}

De Jong, J., 1978. Dry storage and subsequent recovery of cut gerbera flowers as an aid in selection for longevity. Scientia Horticulture 9, 389-397.

Dimitrios, P.N., Tzanetos, I.C., Georgia, P.N., Nikos, P., 2008. A portable sensor for the rapid detection of naphthalene acetic acid in fruits and vegetables using stabilizer in air lipid films with incorporated auxinbinding protein 1 receptor. Talanta 77(2), 786-792.

Droon, W.G., 1997. Water relations of cut flowers. Horticulture Review 18, 1-85.

Dumitras, A., Lazar, V., Zaharia, D., Cantor, M., 2002. Influence of some domestic preserving solutions on the vase life time of some flower species. Buletinul Universitatii de Stiinte Agricole si Medicina Veterinara Cluj Napoca Seria Horticultura 57, 142-145.

Halevy, A.H., Mayak, S., 1979. Scenescence and postharvest physiology of cut flowers. In: Horticulture Review 1, 204-236.

Halevy, A.H., Mayank.1981. Scenescence and post-harvest physiology of cut flowers, Part 2, Horticulture Review 3, 59-143.

Jowkar, M.M., 2007. Biocide effect on cut tuberose cv. Gol Dorosh t-e-Mahallat vase solution microbial kind and population. Acta Horticulture, 755.

Kumar, V., Battacharjee, S.K., Rajive Kumar, R., Misra, L., Krishnan, P.S., 2003. Post-harvest and quality of tuberose spikes as affected by colouring agents and storage. Journal of Ornamental Horticulture 6(2), 119.

Marousky, F.J., 1969. Vascular blockage, water absorption, stomatal opening and respiration of cut 'Better Times' roses treated with 8-hydroxyquinolene citrate and sucrose. Journal of American Society of Horticultural Science 96, 38-41.

Mekala, P., Ganga, M., Jawaharlal, M., 2012. Artificial colouring of tuberose flowers for value addition. South Indian Horticulture 60, 216-223.

Pand, A., Santosh, K., 2004. Response of floral preservatives on post-harvest quality of gladiolus spike cultivar "Pink Friendship". Advances in Plant Sciences 17(2), 529-532.

Patil, S.D., Dhaduk, B.K., 2007. Post-harvest behaviour of candytuft cut flowers (Iberia umbellata L.) as affected by harvesting stages, chemical preservatives and colouring solutions. Asian Journal of Horticulture 2(2), 256-265.

Richmond, A.E., Lang, A., 1957. Effect of kinetin on protein content and survival of detached Xanthium leaves. Science 125, 650-651.

Sacher, J.A., 1973. Senescence and post harvest physiology. Annual Review of Plant Physiology 24, 197-310.

Saifuddin, M., Houssain, A.B. M.S., Normanzia, O., Nasrulhaq Boyce, A., Moneruzzaman, K.M., 2009. The effect of Naphthalene acetic acid and Gibberellic acid in prolonging bract longevity and delaying discoloration of Bougainvillea spectabilis. Biotechnology 8(3), 343350.

Salisbury, F.B., Ross, C.W., 1986. Plant physiology. Wadworth, California, 319-329.

Sambandhamurthy, S., Appavu, K., 1980. Effect of the chemicals on the colouring of tuberose (Polianthes tuberosa L.). South Indian Horticulture 7(2), 103-104.

Talukdar, M.C., Barooah, L., 2011. Effect of Pulsing and different holding solutions on flower quality and vase life of Tuberose (Polianthes tuberosa L.) cv. Calcutta Double, Journal Indian hill farming 24(1), 31-33. 